\title{
Expression of thioredoxin 1 and peroxiredoxins in squamous cervical carcinoma and its predictive role in NACT
}

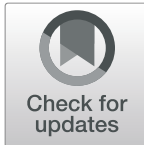

Haiyan Zhu ${ }^{1 \dagger}$, Xuejiao Tao ${ }^{1 \dagger}$, Lulu Zhou ${ }^{1}$, Bo Sheng $^{1}$, Xuejie Zhu $^{2^{*}}$ and Xueqiong Zhu ${ }^{{ }^{*}}$ (I)

\begin{abstract}
Background: This study aims to investigate the expression of thioredoxin 1, peroxiredoxin 1 and peroxiredoxin 2 in bulky cervical squamous carcinoma and its predictive role in cisplatin-based neoadjuvant chemotherapy.

Methods: Initially, the expression of thioredoxin 1, peroxiredoxin 1 and peroxiredoxin 2 protein was analyzed in 13 human cervical squamous cancer tissues and their paired adjacent non-cancerous tissues by western-blotting and immunohistochemistry. Then, correlation between the expression of thioredoxin 1, peroxiredoxin 1, peroxiredoxin 2 and responses to cisplatin-based neoadjuvant chemotherapy was analyzed in 35 paired tumor samples (pre- and post-chemotherapy) from bulky cervical squamous cancer patients by immunohistochemistry.

Results: A clinical response occurred in 48.6\% (17/35) of patients, including 14.3\% (5/35) with a complete response and 34.3\% (12/35) with a partial response. The expression of thioredoxin 1, peroxiredoxin 1 and peroxiredoxin 2 was much higher in cervical squamous cancer tissues compared with paired adjacent non-cancerous tissues by westernblotting and immunohistochemistry. Additionally, the expression of thioredoxin 1, peroxiredoxin 1 and peroxiredoxin 2 was significantly up-regulated in post-chemotherapy tissues compared to pre-chemotherapy cervical cancer tissues. High levels of thioredoxin 1, peroxiredoxin 1 and peroxiredoxin 2 were associated with a poor chemotherapy response in cervical squamous cancer patients.

Conclusions: Thioredoxin 1, peroxiredoxin 1 and peroxiredoxin 2 are frequently over-expressed in cervical squamous cancer. High expression levels of these proteins were related to a poor response to cisplatin-based neoadjuvant chemotherapy. The present study is the first report that thioredoxin peroxidase system may serve as a prediction of the responses to neoadjuvant chemotherapy in cervical squamous cancer.
\end{abstract}

Keywords: Thioredoxin 1, Peroxiredoxin 1, Peroxiredoxin 2, Neoadjuvant chemotherapy, Cervical squamous cancer

\section{Highlights}

1. Thioredoxin 1, peroxiredoxin $1 / 2$ are overexpressed in cervical squamous cancer.

2. High levels of thioredoxin 1 and peroxiredoxin $1 / 2$ related to a poor chemotherapy response in cervical squamous cancer patients.

\footnotetext{
* Correspondence: zxj1022@163.com; zjwzzxq@163.com

${ }^{\dagger}$ Haiyan Zhu and Xuejiao Tao contributed equally to this work.

${ }^{2}$ Department of Gynecology, the First Affiliated Hospital of Wenzhou Medical University, Shangcaicun Road, Wenzhou 325000, China

'Department of Obstetrics and Gynecology, the Second Affiliated Hospital of Wenzhou Medical University, No. 109 Xueyuan Xi Road, Wenzhou 325027, Zhejiang, China
}

3. Thioredoxin peroxidase system may serve as a prediction of the responses to neoadjuvant chemotherapy in cervical squamous cancer.

\section{Background}

The widespread uptake of screening for the prevention and early detection of cervical cancer has been largely successful in more developed countries. However, cervical cancer remains the second most commonly diagnosed cancer and third leading cause of cancer death among females in less developed countries, where a significant proportion of patients are diagnosed with locally advanced cervical cancer, and often have a poor prognosis [1]. Neoadjuvant chemotherapy (NACT) prior

(c) The Author(s). 2019 Open Access This article is distributed under the terms of the Creative Commons Attribution 4.0 International License (http://creativecommons.org/licenses/by/4.0/), which permits unrestricted use, distribution, and reproduction in any medium, provided you give appropriate credit to the original author(s) and the source, provide a link to the Creative Commons license, and indicate if changes were made. The Creative Commons Public Domain Dedication waiver (http://creativecommons.org/publicdomain/zero/1.0/) applies to the data made available in this article, unless otherwise stated. 
to surgery has been widely used for bulky or locally advanced cervical cancer. In a large majority of these patients, treatment is effective, which can shrink the tumor size, suppress micrometastases, and promote the safety and integrity of surgery [2]. In $20-25 \%$ of cases, however, this neoadjuvant chemotherapy fails to achieve tumor regression [2], furthermore, the prognosis of NACT-refractory patients would become worse due to the delay in curative treatment [3]. Therefore, there is an obvious need to identify and develop novel biomarkers which may aid in predicting chemotherapy responses, and thus to assess the treatment options of individual patients.

Recently, appreciable evidence suggested that some approaches of chemotherapy implicate the stimulation of intracellular reactive oxygen species (ROS) production to eliminate cancer cells [4-6], and thus ROS associated oxidative stress have been considered involvement in the development of chemoresistance [7]. Correspondently, human body produces several anti-oxidants that protect against ROS. Indeed, an increasing body of data suggests thioredoxin 1 ( $\operatorname{Trx} 1$ ) together with peroxiredoxins (Prxs), form the thioredoxin peroxidase system that scavenge peroxides, provide protective effects against ROS-induced damages, thereby reducing the efficacy of the treatment $[8,9]$.

$\operatorname{Trx} 1$ is a $12-\mathrm{kDa}$ protein with redox-active dithiol in the active site -Cys-Gly-Pro-Cys- that is ubiquitously present in the human body [10]. It is a defensive protein induced by various stresses and has anti-oxidative, antiapoptotic and anti-inflammatory effects [9]. Trx 1 plays crucial roles in pathophysiological mechanisms of cancer and aberrant expression of Trx 1 has been detected in many forms of cancers [11], correlating with cancer development, metastasis, progression, survival and resistance to chemotherapy [10]. Trx 1 has been reported involved in paclitaxel -induced drug resistance in ovarian cancer A2780 cells [12] and cisplatin-induced resistance in pancreatic cancer cells [13]. Over-expressed human Trx was responsible for the development of cellular resistance to cispaltin in Jurkat T cells [14]. Until now, no study has evaluated the potential role of Trx 1 in cervical carcinogenesis as well as its implication in resistance to chemotherapy among squamous cervical cancer patients.

$\operatorname{Trx} 1$ exerts its main function as an antioxidant mainly via its interactions with downstream peroxiredoxins [15]. Peroxiredoxins are a ubiquitous family of thiol-dependent peroxidases that catalyse the reduction of $\mathrm{H}_{2} \mathrm{O}_{2}$, and different alkyl hydroperoxides [16]. They are involved in a variety of important cellular functions and up-regulated in many human cancer types, contributing to the development of tumor progression and chemo-resistance [17]. However, whether peroxiredoxins expression correlates with chemoresistance in squamous cervical cancer patients is currently unknown.
In line with the above evidence, we hypothesis that chemotherapeutics can cause ROS, possibly resulting into the increase of antioxidant factors such as Trx1, Prx1 and Prx2, providing protective effects against ROSinduced damages, and therefore contribute to resistance development. In fact, our previous study has detected up-regulation of $\operatorname{Prx} 1$ in squamous cervical cancer tissues after NACT relative to the level before chemotherapy by using proteomics profiling [18]. The aim of the current study is to determine the expression of Trx 1, Prx 1 and Prx 2 in squamous cervical carcinoma and their potential roles in predicting chemoresistance among stage IB2 or IIA2 squamous cervical cancer patients treated with neoadjuvant chemotherapy.

\section{Methods}

\section{Patients and tissue specimens}

All tissue samples were collected from the Second Affiliated Hospital of Wenzhou Medical University after informed written patient consent. This study was approved by the ethical committee of the Second Affiliated Hospital of Wenzhou Medical University and conducted according to the Helsinki declaration. At first, a total number of 13 squamous cervical cancer tissues and adjacent normal tissues were collected between January 2014 and April 2015 for the expression analysis of Trx 1, Prx 1 and Prx 2 by western-blotting and immunohistochemistry. The median age of the 13 patients is 49y (range, 36 74y). These patients are in stage IB1 (10 of 13 patients, 76.9\%) and IIA1 (3 of 13 patients, 23.1\%) with the median diameter of $2.13 \pm 0.12$. Ten cases were grade I, 2 cases were grade II and 1 case was grade III. Stage and grading were according to the International Federation of Gynecology and Obstetrics (FIGO) system. Paired tumor cervical cancer tissues and adjacent normal tissues from each patient were obtained after radical hysterectomy.

Another cohort (cohort 2) consisted of 35 patients with IB2 or IIA2 (bulky, primary tumor $>4 \mathrm{~cm}$ in diameter) squamous cervical cancer treated with NACT at the Second Affiliated Hospital of Wenzhou Medical University between January 2007 and August 2014. Clinicopathological variables were recorded, including age at diagnosis, stage, tumor grade and size. Details of treatment was documented for retrospective analysis. Clinicopathologic data was detailed in Table 1 . None of these patients had received chemotherapy, immunotherapy, hormonal therapy or radiotherapy before the specimen collection. Paired tumor samples from each patient were obtained during cervical biopsy (pre-chemotherapy) or surgery (post-chemotherapy). Hematoxylin and eosinstained slides of the cervical tumor from each patient were reviewed for confirmation of pathological features, as well as to select suitable tissue blocks for immunohistochemistry analysis. 
Table 1 Clinical characteristics of cervical squamous cancer patients

\begin{tabular}{llll}
\hline Parameter & $\begin{array}{l}\text { Responders } \\
(n=17)\end{array}$ & $\begin{array}{l}\text { Non-responders } \\
(n=18)\end{array}$ & $P$ \\
\hline $\begin{array}{l}\text { Median age (years) } \\
\text { FIGO stage }\end{array}$ & $47 \pm 8$ & $44 \pm 9$ & $\mathrm{NS}^{*}$ \\
$\quad$ IB2 & $8(\mathrm{IB} 2)$ & $11(\mathrm{IB} 2)$ & $\mathrm{NS}^{*}$ \\
IIA2 & 9 (IIA2) & 7 (IIA2) & \\
$\begin{array}{l}\text { Differentiation } \\
\text { G1 }\end{array}$ & 6 & 7 & $\mathrm{NS}^{*}$ \\
G2 & 10 & 10 & \\
$\quad$ G3 & 1 & 1 & $\mathrm{NS}^{*}$ \\
Primary tumor size $(\mathrm{cm})$ & $4.53 \pm 1.24$ & $4.17 \pm 1.41$ & \\
\hline
\end{tabular}

${ }^{*} P>0.05$, Comparison between the responder and non-responder cohorts

\section{NACT and therapeutic effect evaluation}

All eligible patients received one or two courses of cisplatin-based NACT, as previous described [19]: cisplatin, $60 \mathrm{mg} / \mathrm{m}^{2}$ on day $1 ; 5$-fluorouracil, $750 \mathrm{mg} / \mathrm{m}^{2}$ on day 1 ; mitomycin $\left(8 \mathrm{mg} / \mathrm{m}^{2}\right)$ on day 1 for one or two courses, every 28 days. All of these chemotherapeutics were administrated via uterine artery injection. The chemotherapy response was evaluated by measuring the tumor's two dimensions (the longest diameter and its perpendicular diameter) with magnetic resonance imaging or other radiographic means (CT scan and ultrasound). Eighteen cases $(51.4 \%)$ were measured by MRI, 4 cases (11.4\%) were measured by CT scan and 13 cases (37.2\%) were measured by ultrasound. The chemotherapy response was determined two weeks after completion of neoadjuvant chemotherapy, according to the WHO criteria [20]. Complete response (CR) was defined as the complete remission of the tumor. Partial response (PR) was defined as least a 50\% decrease in the tumor volume. Stable disease (SD) meant a steady state or a response less than 50\%, and progressive disease (PD) was defined as an unequivocal increase of at least $25 \%$ in the tumor volume. The patients with CR or PR were defined as chemotherapy responders, while the patients with SD or PD were deemed as chemotherapy non-responders. We evaluated the response after the first cycle chemotherapy, if the result showed response, we then did the second one. Totally, there are 18 cases under-going one cycle and 17 cases under-going two cycles. All patients were treated with radial hysterectomy and bilateral pelvic lymphadenectomy two to three weeks after completion of the NACT regimen as described previously [19].

\section{Western blotting}

Samples were homogenized and lysed in Laemmli buffer with a cocktail of protease inhibitors. The total protein concentrations were quantified by the bicinchoninic acid protein assay (Thermo Scientific, Rockford, IL). Equal amounts of total protein were resolved by sodium dodecyl sulfate- polyacrylamide gel electrophoresis, transferred to a nitrocellulose membrane under constant voltage and blocked with tris buffered saline with tween (TBST) containing 5\% non-fat dried milk. Primary antibodies and secondary antibodies were diluted in TBST and applied with a washing step in between. Proteins were detected using the Amersham ECL western blotting detection kit (GE Healthcare, Piscataway, NJ). Primary antibodies used including: anti-Trx 1 (CST, USA; 1:1000), anti-Prx 1(Abcam, USA; 1:2000), anti- Prx 2 (Abcam, USA; 1:1000), anti- $\beta$-actin (Abcam, USA; 1 : $5000)$. Image J software was used to quantify western blot bands in this study. Image $\mathrm{J}$ is a Java-based image analysis package widely used in quantitating visual results such as bands on gels or photomicrographs.

\section{Immunohistochemistry}

Formalin-fixed paraffin embedded tissues were cut into sections of $4 \mu \mathrm{m}$ and then mounted onto poly-L-lysinecoated slides. In briefly, after de-paraffinized in xylene and rehydrated through graded ethanol solutions, sections were boiled in a $10 \mu \mathrm{mol} / \mathrm{L}$ citric buffer suolution (pH 6.0) in a microwave oven for $10 \mathrm{~min}$, followed by incubation with $3 \%$ hydrogen peroxide in methanol to suppress the endogenous peroxidase activity and overnight incubation with primary antibodies (Trx1, CST, USA; 1:200; Prx1, Abcam, USA; 1:200, Prx 2, Abcam, USA; $1: 200)$ at $4{ }^{\circ} \mathrm{C}$. Subsequently, the sections were incubated with pre-diluted secondary antibody (Santa Cruz, USA) for $2 \mathrm{~h}$ at room temperature, followed by further incubation with 3,3-diaminobenzidine tetrahydrochloride. Finally, the slides were counterstained with hematoxylin. Appropriate positive and negative controls were stained in parallel. For negative controls, primary antibodies were replaced with phosphate-buffered saline solution. Human cervical cancer tissues were used as a positive control.

\section{Evaluation of immunoreactivity}

Trx 1, Prx 1 and Prx2 protein were detected primarily in cytoplasm and partially in cytomembrane. Assessment of expression of Trx1, Prx-1 and Prx2 staining was determined as previously described [19]. In brief, expression of the these markers was determined by an individual labeling score considering percent and staining intensity of positive cells by two independent assessors blinded to the study end points. [21] Intensity of stained cells was graded semi-quantitatively into four levels as following: 0 (no staining); 1 point (weak staining: light yellow); 2 points (moderate staining: yellow brown) and 3 points (strong staining: brown). The percentage was scored as following: 0 ( 0 to $5 \%$ ), 1 point ( 6 to $24 \%$ ), 2 points (25 to $49 \%$ ), 3 points (50 to $74 \%$ ), and 4 points (75 to $100 \%$ ). 
Intensity and fraction of positive cell scores were multiplied for each marker and thus got the immunoreactive score.

\section{Statistical analysis}

The Kolmogorov-Smirnov test of normality was applied. Continuous variables were presented as mean \pm standard deviations and non-normally distributed variables were presented as median (P25- P75). Since the patient profile between the NACT responsive and non-responsive group displayed a normal distribution, a Student t-test (age and tumor size) or chi-square test (FIGO stage and Histological grade) was used for analysis. Protein expression between the two groups displayed a non-normal distribution and was evaluated by non-parametric tests such as the Wilcoxon test. The software of SPSS 16.0 (SPSS Inc., IL) was used for statistical analysis. A $P$-value less than 0.05 were considered statistically significant.

\section{Results}

\section{Patient characteristics}

The first cohort consisted 13 bulky squamous cervical cancer patients. The median age was 49y (range $36 \sim 74 \mathrm{y}$ ), and stage IB1 and stage IIA1 was 76.9 and $23.1 \%$, respectively.

The second cohort consisted 35 patients with bulky stage IB2 IIA2 squamous cervical cancer treated with NACT. The median age was $44 y$ (range $25 \sim 63 y$ ). Nineteen tumors were stage IB2 (54.3\%), and sixteen cases were stage IIA2 $(45.7 \%)$. Of the 35 patients identified, 5 patients $(14.3 \%)$ showed a complete response and 12 patients (34.3\%) showed a partial response, while 13 patients $(37.1 \%)$ had a stable disease and other five patients (14.3\%) showed progressive disease. Thus, 17 cases were chemo-sensitive (48.6\%) and 18 were chemo-resistant (51.4\%). There were no significant differences in patients or tumor characteristics between the responder and non-responder cohorts (Table 1). Clinicopathologic characteristics are shown in Table 1.

\section{Trx 1, Prx1 and Prx2 protein were over-expressed in squamous cervical cancer}

Initially, the expression of $\operatorname{Trx} 1, \operatorname{Prx} 1$ and $\operatorname{Prx} 2$ in 13 cases of squamous cervical cancer and adjacent normal tissue was investigated by Western blotting. As shown in Fig. 1, all of these three indicated proteins were overexpressed in squamous cervical cancer tissues compared to adjacent normal tissues. The expression and localization of Trx 1, Prx 1 and Prx 2 in squamous cervical cancer and adjacent normal tissue were further examined by immunohistochemistry. A strong cytoplasmic or membranous expression of Trx 1 was observed in all 13 squamous cervical cancer samples analyzed, and generally limited to tumor cells (Fig. 2). The expression pattern of Prx 1 and Prx 2 in squamous cervical cancers was similar to Trx 1 . Interestingly, with regard to adjacent normal tissue, moderate staining of Trx 1 was observed in superficial layer cells and intermediate layer cells of cervical epithelial among all the 13 samples, while only three cases showed positive staining in basal layer cells, in which two cases showed weak staining and the third case showed moderate staining. Similarly, all of the adjacent normal tissues (13/13, 100\%) showed weak or moderate staining for Prx 1 and Prx 2 in superficial layer and intermediate layer of cervix epithelia, only 3 cases $(23.1 \%)$ expressed weak or moderate staining in basal layer of cervix. Since, cervical epithelial cells in basal cells may be the source of malignant transformed cells, we compared the different expression of these three proteins between cervical cancers samples and the basal layer cells of adjacent normal tissues. Quantification of Trx 1, Prx 1 and $\operatorname{Prx} 2$ staining showed that Trx 1, Prx 1 and Prx 2 immunoreactive scores were much higher in squamous cervical cancer compared to basal layer cells of adjacent normal tissues $(P=0.007, P=0.01, P=0.01$, respectively), (Table 2). Figure 2 illustrates the immunostaining for all markers studied in squamous cervical cancer tissues and adjacent normal tissues.

\section{Trx 1, Prx 1 and Prx 2 protein were upregulated after NACT}

To investigate the effect of chemotherapy on the expression of Trx 1, Prx 1 and Prx 2 in squamous cervical cancer samples, the expression of Trx 1, Prx 1 and Prx 2 was performed in 35 matched primary squamous cervical cancer biopsies before and after cisplatin-based NACT using immunohistochemistry. Pre-chemotherapy cervical cancer tissues consistently showed weak or moderate positive staining of $\operatorname{Trx} 1, \operatorname{Prx} 1$ and $\operatorname{Prx} 2$, while post-chemotherapy tissue consistently showed moderate or intense positive staining (Fig. 3). Using a Wilcoxon test, the expression of $\operatorname{Trx} 1, \operatorname{Prx} 1$ and Prx 2 were significantly stronger in post-chemotherapy tissues compared to pre-chemotherapy tissues, $(P=0.000, P=$ $0.001, P=0.010$, respectively) (Table 3 ), suggesting Trx 1 , Prx 1 and Prx 2 protein were implicated in squamous cervical tumor responses to chemotherapy.

\section{Increased expression of Trx 1, Prx1 and Prx2 was associated with chemoresistance}

To explore the role of $\operatorname{Trx} 1, \operatorname{Prx} 1$ and $\operatorname{Prx} 2$ in the development of chemo-resistance, the expression of Trx 1, Prx 1 and Prx 2 in pre-chemotherapy cervical cancer tissues between chemotherapy responders and non-responders was examined by immunohistochemistry. As shown in Table 4, the expression of $\operatorname{Trx} 1, \operatorname{Prx} 1$ and Prx 2 in pre-chemotherapy cervical cancer tissues was markedly stronger in chemotherapy non-responders than that in responders, suggesting patients with high 


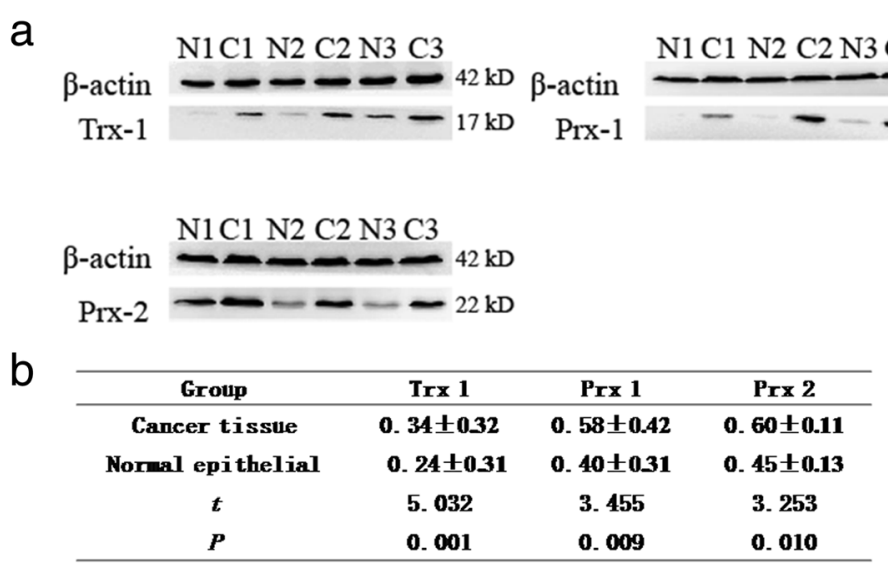

Fig. 1 Western blot analysis of Trx 1, Prx 1 and Prx 2 expression in cervical squamous cancer and adjacent normal tissue. (a, b) Trx 1, Prx 1 and Prx 2 protein expression were markedly up-regulated in squamous cervical cancer tissues compared to adjacent normal tissues. Actin protein level was used to validate equal sample loading

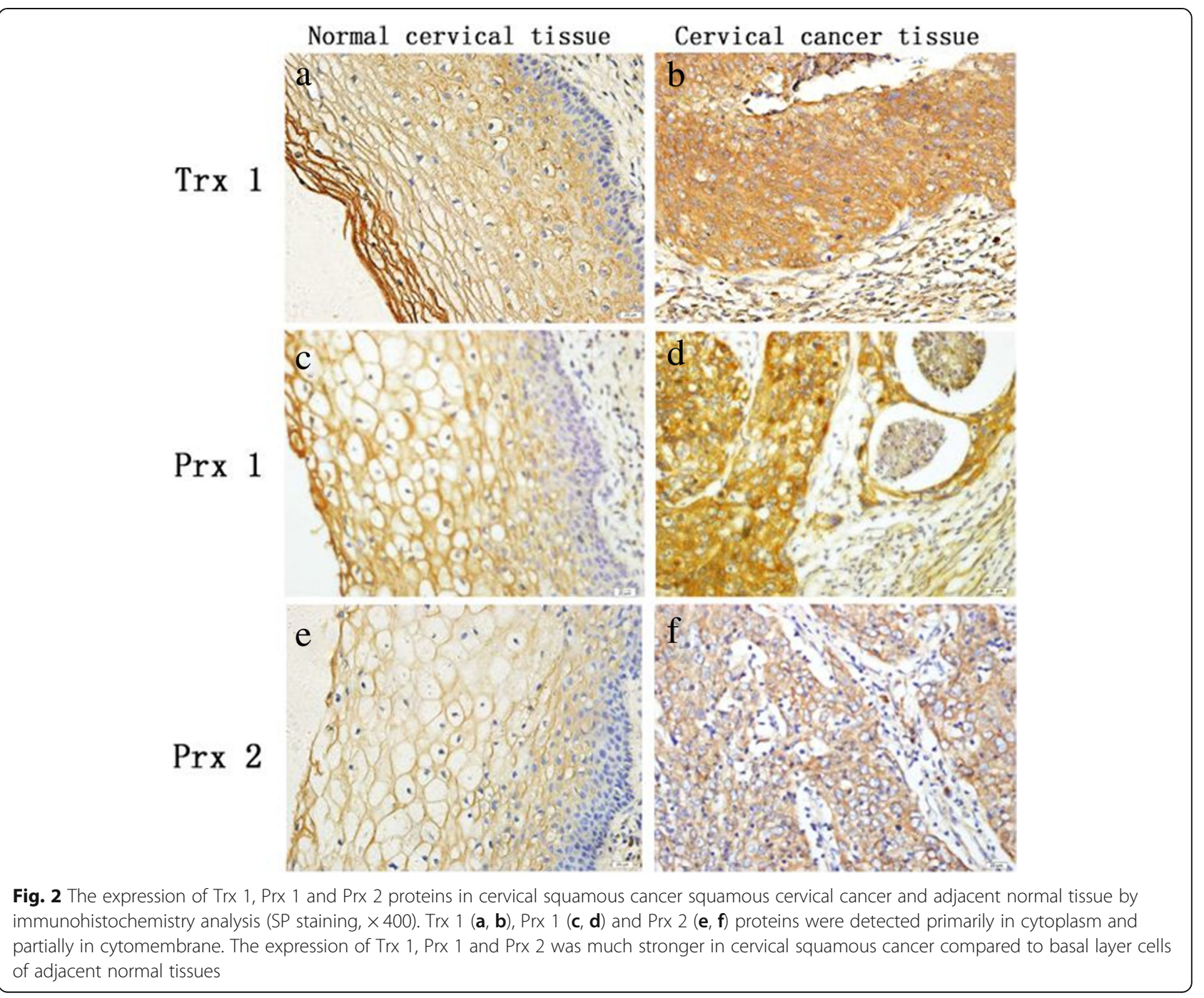


Table 2 The expression of Trx 1, Prx 1 and Prx 2 proteins in tumor cells of cervical cancer and the basal layer cells of adjacent normal tissues

\begin{tabular}{lllll}
\hline Group & $\mathrm{n}$ & $\operatorname{Trx} 1$ & $\operatorname{Prx} 1$ & $\operatorname{Prx} 2$ \\
\hline Cancer cell & 13 & $10.5(8.00-12.00)$ & $7.0(4.00-8.00)$ & $10.5(8.00-12.00)$ \\
Normal basal cell & 13 & $0.0(0.00-3.25)$ & $0.0(0.00-3.50)$ & $2.0(0.00-6.00)$ \\
$Z$ & & -2.694 & -2.578 & -2.561 \\
$P$ & 0.007 & 0.01 & 0.01 \\
\hline
\end{tabular}

Data are expressed as median (P25-P75)

${ }^{*} P<0.05$

levels of Trx 1, Prx1 and Prx2 were more resistant to cisplatin-based NACT than those with low protein expression $(P<0.05$, respectively).

\section{The correlation between Trx 1 and Prx $1 / 2$ in squamous cervical cancer}

The correlation between Trx 1 and Prx $1 / 2$ in 13 cervical squamous cancer samples without chemotherapy and 35 pre-chemotherapy cervical squamous cancer samples were further explored. Interestingly, our results showed that there was a positive correlation between Trx 1 and Prx 2, but not between Trx 1 and Prx 1, suggesting Trx
1 and Prx 2 may function together in cervix carcinogenesis and chemoresistance (Table 5).

\section{Discussion}

Aberrant reactive oxygen species levels has been well document to the initiation and progression of cancer. Numerous types of cancer cells are characterized by an increase in reactive oxygen species production, and often exhibit an altered redox environment compared with normal cells $[7,16]$. Trx 1 is essential for the protection against oxidative stress, for the maintaining the balance of the cellular redox status and for the regulation of differentiation and cell fate [22]. This wide range of cellular functions leads into its involvement in a variety of diseases, including cancer. In fact, an increased level of Trx 1 is found in many primary human cancers, which may contribute to the development of tumor progression [11]. Trx 1 can stimulate tumor cell proliferation, inhibit apoptosis, promote tumor growth and decrease cancer patient survival [10]. Consistent with its functional role in other cancers, in the current study, we detected, for the first time, an increased expression of $\operatorname{Trx} 1$ in

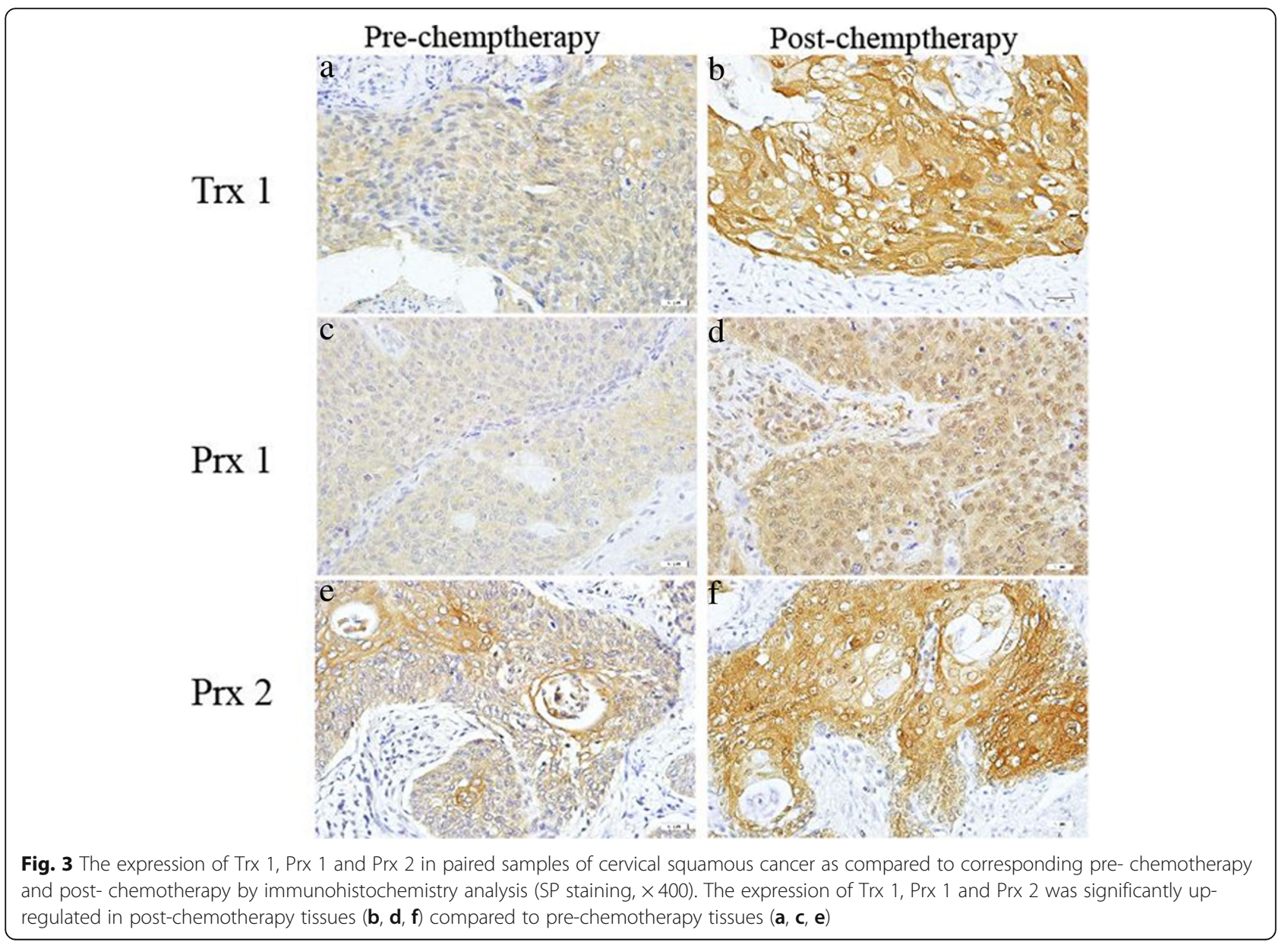


Table 3 The expression of Trx 1, Prx 1 and Prx 2 in cervical cancer cells pre- and post- chemotherapy

\begin{tabular}{lllll}
\hline & $\mathrm{n}$ & Trx 1 & Prx 1 & Prx 2 \\
\hline Pre-chemotherapy & 35 & $6.0(4.00-8.00)$ & $6.0(4.00-8.00)$ & $8.0(5.50-8.25)$ \\
Post-chemotherapy & 35 & $9.0(8.00-12.00)$ & $8.0(8.00-12.00)$ & $12.0(8.00-12.00)$ \\
$Z$ & & -3.973 & -3.215 & -3.313 \\
$P$ & & $0.000^{*}$ & $0.001^{*}$ & $0.010^{*}$ \\
\hline
\end{tabular}

Data are expressed as median (P25-P75)

$* P<0.05$

squamous cervical caners, suggesting Trx 1 may play an important role in the development of cervical cancer.

Trx 1 exerts most of its antioxidant properties through peroxiredoxins [22]. Trx 1 reduces the oxidized form of Prxs, and reduced Prx in turn scavenges ROS, such as $\mathrm{H}_{2} \mathrm{O}_{2}$. Similar to Txr 1, increased levels of Prxs have been demonstrated in many human cancers, including cervical cancer. Prx 1 has been reported to exert its tumor- promoting function in numerous types of human cancer, and appears to be mediated through its interaction with several cancer-associated signal pathways, such as increasing the expression of vascular endothelial growth factor and activating c-Jun and AP-1, inhibiting epithelial-mesenchymal transition through the inhibition of E-cadherin and suppressing apoptosis through the inhibition of apoptosis signal-regulating kinase 1 activity [16]. Increased expression levels of $\operatorname{Prx} 2$ have been found in breast prostate cancer, in prostate, and in esophageal cancer [16]. With respect to cervical cancer, Prx 2 protein levels were found to be progressively upregulated from normal tissue to cervical intraepithelial neoplasia (CIN1, CIN2, and CIN3), and cervical cancer $[23,24]$. In agreement with previous literature, we found that $\operatorname{Prx} 1$ and Prx 2 protein were over-expressed in squamous cervical caners compared with basal layer of normal cervical tissues, suggesting a possible role for Prx $1 / 2$ as a tumor promoter in cervical cancer. Collectively, these results supported the notion that cervical cancer cells, compared with normal cells, had higher reactive oxygen species levels as by-products of their metabolism. To survive from this redox status, the levels of thioredoxin peroxidase system, including Trx 1, Prx 1 and Prx 2 , were up-regulated. The mechanism by which this

Table 4 The expression of Trx 1, Prx 1 and Prx 2 in cancer cells of pre-chemotherapy cervical cancer between chemotherapyresponse and non-response group

\begin{tabular}{lllll}
\hline Group & $\mathrm{n}$ & $\operatorname{Trx} 1$ & $\operatorname{Prx} 1$ & $\operatorname{Prx} 2$ \\
\hline Response & 17 & $4.0(4.00-8.00)$ & $6.0(4.00-8.00)$ & $8.0(4.00-8.00)$ \\
Non-response & 18 & $8.0(4.50-8.00)$ & $8.0(8.00-12.00)$ & $8.0(6.50-11.25)$ \\
$Z$ & & -2.199 & -2.364 & -2.024 \\
$P$ & & $0.028^{*}$ & $0.018^{*}$ & $0.043^{*}$ \\
\hline
\end{tabular}

Data are expressed as median (P25-P75)

${ }^{*} P<0.05$ system exerts its oncogenic action in cervical cancer is still poorly understood, and further studies are required.

As mentioned above, cervical squamous cancer cells may display variable profiles with respect to their redoxgenerating and -adaptive systems, and this unique capability of cancer cells may contributed to the development of resistance to chemotherapy, as these treatments are strongly dependent on ROS-induced cytotoxicity [4]. Recently, Trx 1 was widely documented contribution to the development to chemo-resistance. Trx 1 was found to be up-regulated in paclitaxel-resistant ovarian cancer A2780 cells and bortezomib-resistant multiple myeloma cells compared with their parental cells. [12, 25]. Inhibition of Trx 1 by small interfering RNA or a Trx 1 inhibitor can markedly sensitize diffuse Large B cell lymphomas cells to doxorubicin-induced cell growth inhibition [26] and enhance the growth inhibitory actions of cisplatin in pancreatic ductal adenocarcinoma [13]. Trx 1 promoted paclitaxel-induced drug resistance by increasing FOXO1 transcriptional activity and suppressing drug-induced apoptosis in ovarian cancer A2780 cell [12]. Additionally, Trx is downstream of Smad7 in a pathway that confers a growth advantage to pancreatic cancer cells and that increases their resistance to cisplatin-mediated apoptosis [13]. Our current study found that $\operatorname{Trx} 1$ was markedly up-regulated after chemotherapy, suggesting $\operatorname{Trx} 1$ involved in chemotherapy response in cervical squamous cancer. Moreover, patients with high levels of $\operatorname{Trx} 1$ were more resistant to cisplatin-based NACT than those with low protein expression, which supported the recently highlighted roles of $\operatorname{Trx} 1$ in the development of chemoresistance.

In addition to $\operatorname{Trx} 1$, an increasing body of evidence demonstrates that elevated levels of Prx 1 and Prx 2 are often associated with chemoresistance to various drugs.

Table 5 The relevance between Trx 1 and Prx 1/2 in tumor cells of cervical squamous cancer

\begin{tabular}{llll}
\hline Group & $\mathrm{n}$ & Trx 1 & \\
\cline { 3 - 4 } & & $\mathrm{r}$ & $\mathrm{P}$ \\
\hline $\operatorname{Prx} 1$ & 48 & 0.04 & 0.79 \\
$\operatorname{Prx} 2$ & 48 & 0.31 & $0.04^{*}$ \\
\hline${ }^{*} P<0.05$ & & &
\end{tabular}


High levels of Prx 1 and $\operatorname{Prx} 2$ correlated with cisplatin chemoresistance in gastric cancer SNU638 cells [27], in human erythroleukemia K652, in human breast cancer MCF-7 cells [28], and in human ovarian carcinoma SKOV-3 cells [28], as increased levels of this antioxidant inhibited apoptosis. Elevated Prx 1 induced resistance to docetaxel treatment through suppression of FOXO1-induced apoptosis in lung cancer A549 xenograft tumors [29]. Furthermore, in gastric carcinoma cells, Prx 2-specific antisense vectors restored the induction of proapoptotic pathways following cisplatin treatment, confirming the important role of $\operatorname{Prx} 2$ in the resistance process [30]. Our previous study detected up-regulation of Prx 1 in squamous cervical cancer tissue after NACT relative to the level before chemotherapy by using proteomics profiling [15]. In the current study, our data provided evidence that high expression of $\operatorname{Prx} 1$ and $\operatorname{Prx} 2$ was significantly correlated with a poor response to chemotherapy in squamous cervical cancer. These findings re-confirm our notion that thioredoxin peroxidase system confers the development of drug resistance. Chemo-resistant cervical cancer has higher levels of Trx 1 and Prxs, which hamper the therapeutic efficacy by scavenging the ROS involved in the mechanism of chemotherapeutic drugs.

As with any retrospective study, these are some limitation in this study. At first, response was not analyzed pathologically, which providing more information on the remaining cells and stroma regarding the expression of the proteins of interest. Secondly, the chemotherapy response was evaluated by three radiographic means (magnetic resonance imaging, CT scan and ultrasound), which may cause heterogeneity. In addition, this retrospective study, because of its nature that may have not well assessed the response and the different number of chemo cycles, suggest that could be an association between higher basal or prechemotherapy expression of these proteins with either the response or the number of chemo cycles.

\section{Conclusions}

In conclusion, our current study detected an increased level of $\operatorname{Trx} 1$, Prx 1 and $\operatorname{Prx} 2$ in cervical squamous cancer, suggesting a possible role for thioredoxin peroxidase system as a tumor promoter in cervical squamous cancer. Furthermore, our results demonstrated that the higher expression of $\operatorname{Trx} 1$, Prx 1 and $\operatorname{Prx} 2$ in prechemotherapy could predict a poor response to NACT in cervical squamous cancer. Measuring Trx 1, Prx 1 and Prx 2 in biopsy samples before treatment may contribute to more effective management of bulky cervical cancer. More studies with large samples are needed to determine the predictive role of $\operatorname{Trx} 1, \operatorname{Prx} 1$ and $\operatorname{Prx} 2$ in cervical cancer chemotherapy.

\begin{abstract}
Abbreviations
CIN: Cervical intraepithelial neoplasia; CR: Complete response:

FIGO: International Federation of Gynecology and Obstetrics;

NACT: Neoadjuvant chemotherapy; PD: Progressive disease; PR: Partial response; Prxs: Peroxiredoxins; ROS: Reactive oxygen species; SD: Stable disease; Trx 1: Thioredoxin 1
\end{abstract}

\section{Acknowledgments \\ Not applicable.}

\section{Authors' contributions}

XQZ and XJZ conceived and directed the project. HYZ and XJT acquired and analyzed data; LLZ and BS analyzed and interpreted data; HYZ drafted the work or substantively revised it. All authors read and approved any substantially modified version and the final manuscript.

\section{Funding}

This work was supported by grants from international cooperation project of Wenzhou city (NO. H20150003) and Health and Family Planning Commission of Zhejiang Province (NO. 2017KY485). Additional support was provided by Key Lab of Wenzhou city-Gynecological Oncology. The funding bodies had no role in the design of the study and collection, analysis, and interpretation of data and in writing the manuscript.

\section{Availability of data and materials}

The datasets used and/or analyzed during the current study available from the corresponding author on reasonable request.

\section{Ethics approval and consent to participate}

This study was approved by the ethical committee of the Second Affiliated Hospital of Wenzhou Medical University and conducted according to the Helsinki declaration. All tissue samples were collected from the Second Affiliated Hospital of Wenzhou Medical University after informed written patient consent

\section{Consent for publication}

Not applicable. This manuscript does not contain any individual persons data.

\section{Competing interests}

The authors declare that they have no competing interests.

Received: 4 April 2018 Accepted: 16 August 2019

Published online: 30 August 2019

\section{References}

1. Torre LA, Bray F, Siegel RL, Ferlay J, Lortet-Tieulent J, Jemal A. Global cancer statistics, 2012. CA Cancer J Clin. 2015;65(2):87-108.

2. He L, Wu L, Su G, Wei W, Liang L, Han L, Kebria M, Liu P, Chen C, Yu Y, et al. The efficacy of neoadjuvant chemotherapy in different histological types of cervical cancer. Gynecol Oncol. 2014;134(2):419-25.

3. Jin L, Shen $Q$, Ding S, Jiang W, Jiang L, Zhu X. Immunohistochemical expression of Annexin A2 and S100A proteins in patients with bulky stage IB-IIA cervical cancer treated with neoadjuvant chemotherapy. Gynecol Oncol. 2012;126(1):140-6.

4. Filippova M, Filippov V, Williams VM, Zhang K, Kokoza A, Bashkirova S, Duerksen-Hughes $P$. Cellular levels of oxidative stress affect the response of cervical cancer cells to chemotherapeutic agents. Biomed Res Int. 2014; 2014:574659.

5. Marinescu S, Anghel R, Gruia MI, Beuran M. Involvement of reactive oxygen species in the mechanisms associated with cervical cancer specific treatment. Chirurgia. 2014;109(6):806-11.

6. Zhu H, Luo H, Zhang W, Shen Z, Hu X, Zhu X. Molecular mechanisms of cisplatin resistance in cervical cancer. Drug Des Devel Ther. 2016;10: 1885-95.

7. Tong L, Chuang CC, Wu S, Zuo L. Reactive oxygen species in redox cancer therapy. Cancer Lett. 2015;367(1):18-25.

8. Kang SW, Rhee SG, Chang TS, Jeong W, Choi MH. 2-Cys peroxiredoxin function in intracellular signal transduction: therapeutic implications. Trends Mol Med. 2005;11(12):571-8. 
9. Watanabe R, Nakamura H, Masutani H, Yodoi J. Anti-oxidative, anti-cancer and anti-inflammatory actions by thioredoxin 1 and thioredoxin-binding protein-2. Pharmacol Ther. 2010;127(3):261-70.

10. Powis G, Montfort WR. Properties and biological activities of thioredoxins. Annu Rev Biophys Biomol Struct. 2001;30:421-55.

11. Lincoln DT, Ali Emadi EM, Tonissen KF, Clarke FM. The thioredoxinthioredoxin reductase system: over-expression in human cancer. Anticancer Res. 2003;23(3B):2425-33.

12. Wang J, Yang H, Li W, Xu H, Yang X, Gan L. Thioredoxin 1 upregulates FOXO1 transcriptional activity in drug resistance in ovarian cancer cells. Biochim Biophys Acta. 2015;1852(3):395-405.

13. Arnold NB, Ketterer $K$, Kleeff J, Friess $H$, Buchler MW, Korc M. Thioredoxin is downstream of Smad7 in a pathway that promotes growth and suppresses cisplatin-induced apoptosis in pancreatic cancer. Cancer Res. 2004;64(10):3599-606.

14. Sasada T, Iwata S, Sato N, Kitaoka Y, Hirota K, Nakamura K, Nishiyama A, Taniguchi Y, Takabayashi A, Yodoi J. Redox control of resistance to cisdiamminedichloroplatinum (II) (CDDP): protective effect of human thioredoxin against CDDP-induced cytotoxicity. J Clin Invest. 1996;97(10):2268-76.

15. Woolston CM, Storr SJ, Ellis IO, Morgan DA, Martin SG. Expression of thioredoxin system and related peroxiredoxin proteins is associated with clinical outcome in radiotherapy treated early stage breast cancer. Radiother Oncol. 2011:100(2):308-13.

16. Nicolussi A, D'Inzeo S, Capalbo C, Giannini G, Coppa A. The role of peroxiredoxins in cancer. Mol Clin Oncol. 2017;6(2):139-53.

17. Raninga PV, Trapani GD, Tonissen KF. Cross talk between two antioxidant systems, Thioredoxin and DJ-1: consequences for cancer. Oncoscience. 2014;1 (1):95-110.

18. Zou S, Shen Q, Hua Y, Jiang W, Zhang W, Zhu X. Proteomic identification of neoadjuvant chemotherapy-related proteins in bulky stage IB-IIA squamous cervical cancer. Reprod Sci. 2013;20(11):1356-64.

19. Zhu H, Wu J, Zhang W, Luo H, Shen Z, Cheng H, Zhu X. PKM2 enhances chemosensitivity to cisplatin through interaction with the MTOR pathway in cervical cancer. Sci Rep. 2016;6:30788.

20. WHO. Handbook for Reporting Results of Cancer Treatment. Geneva: WHO Offset Publication; 1989. No.48

21. Zhu X, Jin L, Zou S, Shen Q, Jiang W, Lin W, Zhu X. Immunohistochemical expression of RAGE and its ligand (S100A9) in cervical lesions. Cell Biochem Biophys. 2013;66(3):843-50.

22. Mahmood DF, Abderrazak A, El Hadri K, Simmet T, Rouis M. The thioredoxin system as a therapeutic target in human health and disease. Antioxid Redox Signal. 2013;19(11):1266-303.

23. Kim K, Yu M, Han S, Oh I, Choi YJ, Kim S, Yoon K, Jung M, Choe W. Expression of human peroxiredoxin isoforms in response to cervical carcinogenesis. Oncol Rep. 2009;21(6):1391-6.

24. Lomnytska MI, Becker S, Bodin I, Olsson A, Hellman K, Hellstrom AC, Mints $M$, Hellman U, Auer G, Andersson S. Differential expression of ANXA6, HSP27, PRDX2, NCF2, and TPM4 during uterine cervix carcinogenesis: diagnostic and prognostic value. Br J Cancer. 2011;104(1):110-9.

25. Raninga PV, Di Trapani G, Vuckovic S, Bhatia M, Tonissen KF. Inhibition of thioredoxin 1 leads to apoptosis in drug-resistant multiple myeloma. Oncotarget. 2015;6(17):15410-24.

26. Li C, Thompson MA, Tamayo AT, Zuo Z, Lee J, Vega F, Ford RJ, Pham LV. Over-expression of Thioredoxin-1 mediates growth, survival, and chemoresistance and is a druggable target in diffuse large B-cell lymphoma. Oncotarget. 2012;3(3):314-26.

27. Chung YM, Yoo YD, Park JK, Kim YT, Kim HJ. Increased expression of peroxiredoxin II confers resistance to cisplatin. Anticancer Res. 2001;21 (2A):1129-33.

28. Kalinina EV, Berezov TT, Shtil AA, Chernov NN, Glazunova VA, Novichkova $M D$, Nurmuradov NK. Expression of peroxiredoxin 1, 2, 3, and 6 genes in cancer cells during drug resistance formation. Bull Exp Biol Med. 2012 153(6):878-81.

29. Hwang KE, Park C, Seol CH, Hwang YR, Hwang JS, Jung JW, Choi KH, Jeong ET, Kim HR. Elevated prx1 provides resistance to docetaxel, but is not associated with predictive significance in lung cancer. Tuberc Respir Dis. 2013;75(2):59-66

30. Yo YD, Chung YM, Park JK, Ahn CM, Kim SK, Kim HJ. Synergistic effect of peroxiredoxin II antisense on cisplatin-induced cell death. Exp Mol Med. 2002;34(4):273-7.

\section{Publisher's Note}

Springer Nature remains neutral with regard to jurisdictional claims in published maps and institutional affiliations.

\section{Ready to submit your research? Choose BMC and benefit from}

- fast, convenient online submission

- thorough peer review by experienced researchers in your field

- rapid publication on acceptance

- support for research data, including large and complex data types

- gold Open Access which fosters wider collaboration and increased citations

- maximum visibility for your research: over $100 \mathrm{M}$ website views per year

At $\mathrm{BMC}$, research is always in progress.

Learn more biomedcentral.com/submissions 